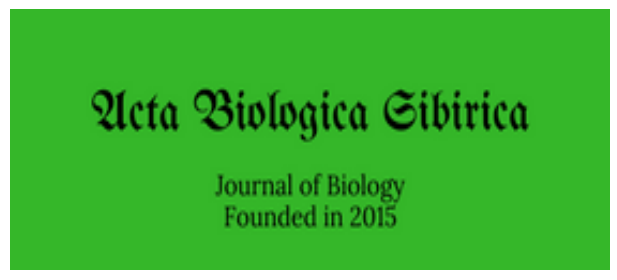

Altai State University

wWw.asu.ru

ISSN 2412-1908

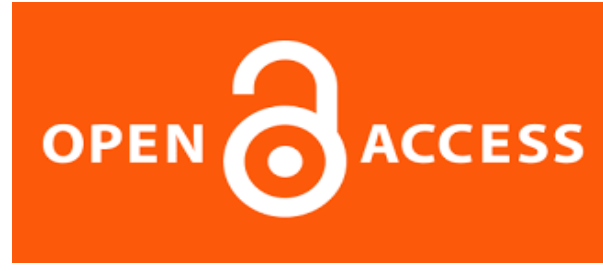

Acta Biologica Sibirica, 2018, 4(1), 6-16

RESEARCH ARTICLE

UDC 595.772

\title{
New records of long-legged flies (Diptera, Dolichopodidae) from Tyva Republic (Russia, Siberia)
}

\author{
I.Ya. Grichanov \\ All-Russian Institute of Plant Protection, Podbelskogo 3, St. Petersburg, Pushkin, 196608 Russia \\ E-mail: grichanov@mail.ru
}

During a 2017 survey conducted in the Tyva Republic of Russia, 322 specimens of Dolichopodidae have been collected. All 36 identified species are recorded for the first time in the Tyva Republic, including two probably undescribed species. Asyndetus longicornis is a new species for Siberia; Mongolian Melanostolus longipilosus and Thinophilus setosus are found for the first time in Russia. In total, 16 genera and 41 (including three unnamed) species are known from Tyva.

Key words: Dolichopodidae; Tyva Republic; new records

\section{Introduction}

Many natural zones of the Earth are present in the Tyva (or Tuva) Republic having several mountain systems. Its biodiversity is shaped by the geographical location in the centre of Asia, between the east-Siberian taiga and central-Asian landscape. Average annual precipitation varies here from $150 \mathrm{~mm}$ (on plains) to 1,000 $\mathrm{mm}$ (in mountains). The summer is moderately warm in mountains, and hot in depressions (about $+25-35^{\circ} \mathrm{C}$ ).

In the case of long-legged flies, the territory of the Tyva Republic remains undercollected, with very few research articles devoted to this land (Negrobov et al., 2013; Grichanov, 2017). The first record of a dolichopodid fly (Medetera signaticornis Loew, 1857) from the Republic was published by V.M. Yanovskii (1976). Medetera asiatica Negrobov et Zaitzev, 1979 was described from this territory (Ishtii-Khem, Ulug-Khemskiy District) (Negrobov, 1979) and reported later from Kyrgyzstan. Negrobov \& Barkalov (2010) described a new species Dolichopus selivanovae from the Altai Mountains including the lakes Muzdy-Bulak, Dzhulukul (Republic of Altai) and Khindiktig-Khol (Tyva). Negrobov et al. (2010) found Hydrophorus zaitzevianus Negrobov, 1976 at the Khindiktig-Khol Lake; the species was described originally from Mongolia.

The material for this study (322 specimens) has been collected by Dr. N.E. Vikhrev, M. Yanbulat (Moscow) and Dr. O.E. Kosterin (Novosibirsk) during a 2017 expedition by standard sweep net mostly. The main collection sites are located in the central Tyva: Piy-Khem Kozhuun (Uyuk River), Kaa-Khem Kozhuun (Kaa-Khem River and Serebryanka River), Tanda Kozhuun (salt Lake Dus-Khol, Lake Khadyn, Mazhalyg River), and Tes-Khem Kozhuun (Eastern Tannu-Ola mountains, TesKhem River). This paper presents the new species records in detail. Records of 36 species listed below are arranged alphabetically by genus. Information on the world distribution for each species listed follows Grichanov (2017). The type localities are provided and the country lists are arranged alphabetically. Material of the newly-recorded species will be deposited at the Zoological Institute of the Russian Academy of Sciences, St. Petersburg, and the Zoological Museum of Moscow State University, Moscow, Russia. Specimens collected by Vikhrev and Yanbulat are mounted on pins and placed in the museum drawers. Specimens collected by Kosterin are kept in vials aggregated in glass cans filled with alcohol. Photos of some habitats for species newly recorded from the Tyva Republic are kindly provided by Oleg Kosterin.

New records of Dolichopodidae

Asyndetus Loew, 1869 
Grichanov, I.Ya. New records of long-legged flies.... Acta Biologica Sibirica, 2018, 4(1), 6-16

\section{Asyndetus longicornis Negrobov, 1973}

MATERIAL. Dus-Khol salt Lake, $700 \mathrm{~m}, 51.36^{\circ} \mathrm{N}, 94.45^{\circ} \mathrm{E}, 2-5$ vii 2017, N. Vikhrev, $1 \delta^{\text {; }}$; Tanda Kozhuun, brackish Lake Khadyn, the western bay, saline, $51.365^{\circ} \mathrm{N}, 94.469^{\circ} \mathrm{E}, 708 \mathrm{~m}$ a.s.l., 2 vii 2017, O. Kosterin, 1 \%.

DISTRIBUTION. Type locality: Mongolia: "Mongolei, Südgobi aimak, 40 km SSO von Nomagon, Salzbodenwiese, in der Nähe von der Wasserquelle". Palaearctic: China (Inner Mongolia), Hungary, Kazakhstan, Mongolia, ?Romania, Russia (Astrakhan), Tajikistan, Turkmenistan, Uzbekistan. New for Siberia.

\section{Campsicnemus Haliday, 1851}

\section{Campsicnemus armatus armatus (Zetterstedt, 1849)}

MATERIAL. Tes-Khem Kozhuun, $16 \mathrm{~km}$ SWS of Samagaltay village, the Tes Khem River right floodplain, grassy poplar forest, $50.470^{\circ} \mathrm{N}, 94.892^{\circ} \mathrm{E}, 1001 \mathrm{~m}$ a.s.l., 4 vii 2017, O. Kosterin, $1 \delta^{\jmath}$.

DISTRIBUTION. Type locality: Denmark: Rosenthal. Gryphium. Palaearctic: Austria, Belgium, Czech, Denmark, Finland, France, Germany, Iceland, Ireland, Italy, Mongolia, Netherlands, Norway, Poland, Russia (Arkhangelsk, Kamchatka, Karelia, Krasnoyarsk, Murmansk, Nenetsia, Ekaterinburg, Tatarstan, Yakutia, Yamalia), Slovakia, Sweden, Turkey (Kars), UK.

\section{Chrysotus Meigen, 1824}

Chrysotus cilipes Meigen, 1824

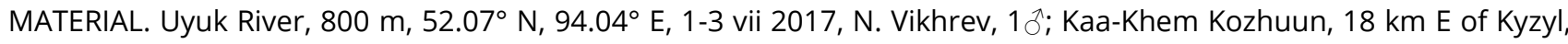
the Kaa-Khem River left bank floodplain at the bridge, larch/poplar forest, $51.706^{\circ} \mathrm{N}, 94.712^{\circ} \mathrm{E}, 639 \mathrm{~m}$ a.s.l., 3 vii $2017, \mathrm{O}$. Kosterin, 3 .

DISTRIBUTION. Type locality: Germany: Hamburg. Palaearctic: Abkhazia, Armenia, Austria, Azerbaijan, Belgium, Bosnia and Herzegovina, Bulgaria, China, Czech Republic, Estonia, Denmark, Finland, France, Germany, Hungary, Ireland, Italy, Japan, Kazakhstan, Korea, Latvia, Lithuania, Luxembourg, Mongolia, Netherlands, Norway, Poland, Portugal Madeira), Romania, Russia (Adygea, Altai Rep., Altai Terr., Astrakhan, Blagoveshchensk, Buryatia, Chita, Irkutsk, KabardinoBalkaria, Karachai-Cherkessia, Karelia, Khabarovsk, Krasnodar, Krasnoyarsk, Moscow, Leningrad, Novgorod, Novosibirsk, Pskov, Rostov, Sakhalin, Tomsk, Vladivostok, Voronezh, Yakutia), Slovakia, Spain, Sweden, Switzerland, Turkey, UK, Ukraine.

\section{Chrysotus gramineus (Fallén, 1823)}

MATERIAL. Kyzyl env., poplar forest, $51.7^{\circ} \mathrm{N}, 94.7^{\circ} \mathrm{E}, 5-7$ vii 2017, N. Vikhrev, 1 ô. $^{\circ}$

DISTRIBUTION. Type locality: not given [Sweden]. Palaearctic: Abkhazia, Andorra, Armenia, Austria, Azerbaijan, Belarus, Belgium, Bosnia and Herzegovina, Bulgaria, China; Czech Republic, Denmark, Estonia; Finland, France, Georgia, Germany, Greece, Hungary, Ireland, Italy, Korea, Kyrgyzstan, Latvia, Lithuania, Moldova, Netherlands, Norway, Poland, Romania, Russia (Adygea, Alania, Altai Rep., Arkhangelsk, Bashkortostan, Blagoveshchensk, Buryatia, Chelyabinsk, Chita, Chukotka, Crimea, Irkutsk, Kabardino-Balkaria, Karachai-Cherkessia, Karelia, Khabarovsk, Kostroma, Krasnodar, Krasnoyarsk, Kursk, Leningrad, Lipetsk, Mordovia, Moscow, Murmansk, Novgorod, Orel, Orenburg, Pskov, Ryazan, Sakhalin, Tatarstan, Tomsk, ?Ural, Vologda, Voronezh, Yakutia, Yaroslavl), Slovakia, Spain, Sweden, Switzerland, Tajikistan, Turkey (Hakkari, Erzurum, Kars), Turkmenistan, UK, Ukraine (Cherkasy, Kharkiv, Kherson).

\section{Dolichopus Latreille, 1796}

\section{Dolichopus brevipennis Meigen, 1824}

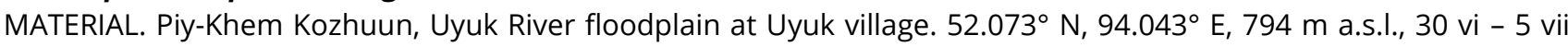

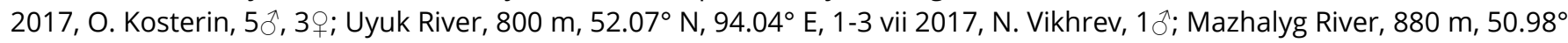
$\mathrm{N}, 95.18^{\circ} \mathrm{E}, 4$ vii 2017, N. Vikhrev, $1 \delta^{\top}$; Tes-Khem Kozhuun, a pass through the Eastern Tannu-Ola Mts., grassy seepage in spruce taiga patch, $50.621^{\circ} \mathrm{N}, 95.193^{\circ} \mathrm{E}, \sim 1440 \mathrm{~m}$ a.s.l., 4 vii 2017 , O. Kosterin, $1 \delta^{\lambda}$.

DISTRIBUTION. Type locality: Sweden. Palaearctic: Austria, Belarus, Belgium, China (Xinjiang), Czech Republic, Denmark, Estonia, Finland, France, Germany, Ireland, Italy, N Kazakhstan, Latvia, Lithuania, Netherlands, Norway, Poland, Russia (Adygea, Altai Rep., Altai Terr., Arkhangelsk, Baskortostan, Buryatia, Irkutsk, Kabardino-Balkaria, Karelia, KhantiaMansia, Krasnodar, Kirov, Komi, Leningrad, Moscow, Murmansk, Novgorod, Omsk, Orenburg, Perm, Pskov, Ryazan, Tomsk, Tyumen, Voronezh, Yaroslavl, Vladivostok, Yakutia), Sweden, UK; Nearctic: Canada and USA: Alaska, Yukon, British Columbia, Northwest Terr., Montana, Alberta, Saskatchewan, Quebec, Prince Edward Is, Newfoundland (Labrador).

\section{Dolichopus calceatus Parent, 1927}

MATERIAL. Kaa-Khem Kozhuun, 18 km E of Kyzyl, Serebryanka River in the Kaa-Khem River left bank floodplain at the bridge, larch/poplar forest, $51.701^{\circ} \mathrm{N}, 94.707^{\circ} \mathrm{E}, 638 \mathrm{~m}$ a.s.l., 6 vii 2017, O. Kosterin, 1 ô, 1 \% .

DISTRIBUTION. Type locality: Russia: "Transbaikale; Pjetschanka, b. Tschita". Palaearctic: China, Kazakhstan, Russia (Altai Rep., Blagoveshchensk, Buryatia, Chita, Irkutsk, Kamchatka, Khabarovsk, Tomsk, Vladivostok, Yakutia).

\section{Dolichopus caligatus Wahlberg, 1850}

MATERIAL. Piy-Khem Kozhuun, Uyuk River floodplain at Uyuk village. 52.073 N, $94.043^{\circ}$ E, 794 m a.s.I., 30 vi - 5 vii 2017, O. Kosterin, 3ð. 
Grichanov, I.Ya. New records of long-legged flies.... Acta Biologica Sibirica, 2018, 4(1), 6-16

DISTRIBUTION. Type locality: "Koonprope Marstrand, Gusum Ostrogothiae" [Sweden]. Palaearctic: Austria, Denmark, Finland, Germany, Lithuania, Netherlands, Norway, Russia (Karelia, Khabarovsk, Leningrad), Sweden, UK.

REMARKS. The species was placed for a long time in synonymy to Dolichopus flavipes Stannius, 1831, was not included into the main keys (Stackelberg, 1930; Negrobov \& Stackelberg, 1969) and was probably recorded from Russia (Irkutsk, Buryatia, Yakutia, Krasnoyarsk, Russian Far East) and Alaska under the name D. flavipes (having type locality Marseille, France).

\section{Dolichopus claviger Stannius, 1831}

MATERIAL. Kyzyl env., poplar forest, 51.7 N, 94.7 E, 5-7 vii 2017, N. Vikhrev, $1 \delta^{\lambda}$; Kaa-Khem Kozhuun, $18 \mathrm{~km}$ E of Kyzyl, Serebryanka River in the Kaa-Khem River left bank floodplain at the bridge, larch/poplar forest, $51.701^{\circ} \mathrm{N}, 94.707^{\circ}$ E, 638 m a.s.l., 6 vii 2017, O. Kosterin, 3ð, 2 \% .

DISTRIBUTION. Type locality: Germany: Hamburg. Palaearctic: Austria, Belarus, Belgium, Bulgaria, Czech, Denmark, Estonia, Finland, France, Germany, Hungary, Ireland, Lithuania, Netherlands, Norway, Poland, Romania, Russia (Adygea, Alania, Altai Terr., Altai Rep., Crimea, Kabardino-Balkaria, Karelia, Karachai-Cherkessia, Krasnodar, Krasnoyarsk, Leningrad, Lipetsk, Magadan, Moscow, Novgorod, Perm, Pskov, Ryazan, Tatarstan, Tomsk, Vologda, Voronezh), Slovakia, Sweden, Switzerland, UK, Ukraine (Cherkasy, Kharkiv, Kyiv), "Yugoslavia".

\section{Dolichopus divisus Becker, 1917}

MATERIAL. Tes-Khem Kozhuun, a pass through the Eastern Tannu-Ola Mts., grassy seepage in spruce taiga patch, $50.621^{\circ} \mathrm{N}, 95.193^{\circ} \mathrm{E}, \sim 1440 \mathrm{~m}$ a.s.l., 4 vii 2017 , O. Kosterin, 1 रे.

DISTRIBUTION. Type locality: Russia: "Urga, Irkutsk". Palaearctic: China, Mongolia, Russia (Irkutsk).

\section{Dolichopus fraterculus Zetterstedt, 1843}

MATERIAL. Piy-Khem Kozhuun, Uyuk River floodplain at Uyuk village. $52.073^{\circ} \mathrm{N}, 94.043^{\circ} \mathrm{E}, 794 \mathrm{~m}$ a.s.l., 30 vi -5 vii 2017, O. Kosterin, 1 ; ; Tanda Kozhuun, salt Lake Dus-Khol, E bank, saline, $51.357^{\circ} \mathrm{N}, 94.446^{\circ} \mathrm{E}, 702 \mathrm{~m}$ a.s.l., 2 vii 2017 , $\mathrm{O}$. Kosterin, 2o; Tes-Khem Kozhuun, a pass through the Eastern Tannu-Ola Mts., grassy seepage in spruce taiga patch, $50.621^{\circ} \mathrm{N}, 95.193^{\circ} \mathrm{E}, \sim 1440 \mathrm{~m}$ a.s.l., 4 vii 2017, O. Kosterin, 1 \%.

DISTRIBUTION. Type locality: Sweden: Jemtlandia meridionali, Asarne. Palaearctic: Finland, Lithuania, Norway, Sweden, Russia (Altai Rep., Karelia, Kuril Is., Magadan, Murmansk, Yamalia, Yakutia); Nearctic: Canada and USA: Alaska, Northwest Terr.

\section{Dolichopus galeatus Loew, 1871}

MATERIAL. Uyuk River, 800 m, $52.07^{\circ}$ N, $94.04^{\circ}$ E, 1-3 vii 2017, N. Vikhrev, $10^{`}$.

DISTRIBUTION. Type locality: Russia: "Sibirien". Palaearctic: Russia (Blagoveshchensk, Buryatia, Irkutsk, Kamchatka, Khabarovsk, Magadan, Yakutia), China (Heilongjiang).

\section{Dolichopus griseifacies Becker, 1917}

MATERIAL. Piy-Khem Kozhuun, Uyuk River floodplain at Uyuk village. $52.073^{\circ} \mathrm{N}, 94.043^{\circ} \mathrm{E}, 794 \mathrm{~m}$ a.s.l., 30 vi -5 vii 2017, O. Kosterin, $6 \hat{\jmath}, 2$.

DISTRIBUTION. Type locality: Russia: "Irkutsk, Sibirien". Palaearctic: Russia (Blagoveshchensk, Buryatia, Irkutsk, Khabarovsk, Vladivostok).

\section{Dolichopus latipennis Fallén, 1823}

MATERIAL. Uyuk River, 800 m, 52.07 N, 94.04 E, 1-3 vii 2017, N. Vikhrev, $1 \delta^{\circ}$.

DISTRIBUTION. Type locality: [Sweden:] "Paroeciae Farhult Scaniae". Palearctic: ?Austria, Belgium, Czech, Denmark, Estonia, Finland, France, Germany, Ireland, Latvia, Netherlands, Norway, Poland, Sweden, UK, N Russia (Karelia, Murmansk, Nenets), E Russia (Altai Rep., Buryatia, Irkutsk, Kamchatka, Novosibirsk, Sakhalin, Yakutia), Mongolia, China (Qinghai); Nearctic: Canada (Quebec, Northwest Terr.).

\section{Dolichopus linearis Meigen, 1824}

MATERIAL. Kaa-Khem Kozhuun, $18 \mathrm{~km}$ E of Kyzyl, Serebryanka River in the Kaa-Khem River left bank floodplain at the bridge, larch/poplar forest, $51.701^{\circ} \mathrm{N}, 94.707^{\circ} \mathrm{E}, 638 \mathrm{~m}$ a.s.l., 6 vii 2017, O. Kosterin, 40․, 7\%; Kaa-Khem Kozhuun, $18 \mathrm{~km} \mathrm{E}$ of Kyzyl, the Kaa-Khem River left bank floodplain at the bridge, larch/poplar forest, $51.706^{\circ} \mathrm{N}, 94.712^{\circ} \mathrm{E}, 639 \mathrm{~m}$ a.s.l., 3 vii 2017, O. Kosterin, 1 3 , 3ㅇ ; Tes-Khem Kozhuun, a pass through the Eastern Tannu-Ola Mts., grassy seepage in spruce taiga patch, $50.621^{\circ} \mathrm{N}, 95.193^{\circ} \mathrm{E}, \sim 1440 \mathrm{~m}$ a.s.l., 4 vii 2017, O. Kosterin, 7 ${ }^{\circ}, 12{ }^{\circ}$; Tes-Khem River, $1000 \mathrm{~m}, 50.47^{\circ} \mathrm{N}, 94.91^{\circ} \mathrm{E}, 4$ vii 2017, N. Vikhrev, $3 \hat{0}$.

DISTRIBUTION. Type locality: not given. Palaearctic: Austria, Belgium, China (Heilongjiang, Jilin, Beijing, Inner Mongolia, Gansu, Xinjiang, Qinghai), Czech Republic, Denmark, Estonia, Finland, France, Georgia, Germany, Hungary, Ireland, Italy, Kazakhstan, Latvia, Mongolia, Netherlands, Norway, Poland, Romania; Russia (Adygea, Altai Rep., Blagoveshchensk, Buryatia, Irkutsk, Kamchatka, Khabarovsk, Khantia-Mansia, Krasnodar, Krasnoyarsk, Leningrad, 
Grichanov, I.Ya. New records of long-legged flies.... Acta Biologica Sibirica, 2018, 4(1), 6-16

Magadan, Novosibirsk, Novgorod, Pskov, Ryazan, Sakhalin, Tatarstan, Vladivostok, Vologda, Voronezh, Yakutia), Slovakia, Sweden, Switzerland, UK.

\section{Dolichopus litorellus Zetterstedt, 1852}

MATERIAL. Piy-Khem Kozhuun, Uyuk River floodplain at Uyuk village. 52.073 N, $94.043^{\circ}$ E, $794 \mathrm{~m}$ a.s.l., 30 vi -5 vii

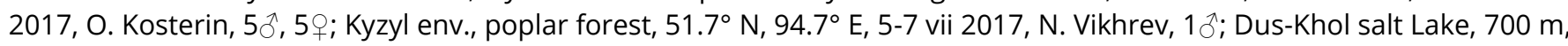
$51.36^{\circ} \mathrm{N}, 94.45^{\circ} \mathrm{E}, 2-5$ vii 2017 , N. Vikhrev, $10^{3}$; Tanda Kozhuun, the Mazhalyg River at the brigde, open grassy banks, $50.981^{\circ} \mathrm{N}, 95.176^{\circ} \mathrm{E}, 882 \mathrm{~m}$ a.s.l., 4 vii 2017 , O. Kosterin, 30 ; Tes-Khem River, $1000 \mathrm{~m}, 50.47^{\circ} \mathrm{N}, 94.91^{\circ} \mathrm{E}, 4$ vii $2017, \mathrm{~N}$. Vikhrev, $10^{\top}$.

DISTRIBUTION. Type locality: Sweden: Lomma Scaniae. Palaearctic: Abkhazia, Austria, Azerbaijan, Belarus, Belgium, Bulgaria, Czech Republic, Denmark, Estonia, France, Germany, Hungary, Iran, Ireland, Italy, Kazakhstan, Mongolia, Netherlands, Poland, Romania, Russia (Altai Rep., Karachai-Cherkessia, Krasnodar, Kursk, Moscow, Omsk, Orel, Pskov, Rostov, Ryazan, Ural, Voronezh, Yakutia), Slovakia, Spain, Sweden, Switzerland, Turkey (Kocaeli-Izmit), UK, Ukraine (Ternopil), Uzbekistan.

\section{Dolichopus longicornis Stannius, 1831}

MATERIAL. Kaa-Khem Kozhuun, $18 \mathrm{~km}$ E of Kyzyl, the Kaa-Khem River left bank floodplain at the bridge, larch/poplar forest, $51.706^{\circ} \mathrm{N}, 94.712^{\circ} \mathrm{E}, 639 \mathrm{~m}$ a.s.l., 3 vii 2017, O. Kosterin, 9 ${ }^{\circ}$, 4우 Tes-Khem Kozhuun, a pass through the Eastern Tannu-Ola Mts., grassy seepage in spruce taiga patch, $50.621^{\circ} \mathrm{N}, 95.193^{\circ} \mathrm{E}, \sim 1440 \mathrm{~m}$ a.s.l., 4 vii 2017, O. Kosterin, $100^{\hat{\prime}}, 7$; Tannu-Ola Mts. pass, $1480 \mathrm{~m}, 50.62^{\circ} \mathrm{N}, 95.16^{\circ} \mathrm{E}, 4$ vii 2017 , N. Vikhrev, $10^{\wedge}$.

DISTRIBUTION. Type locality: not given [Germany: ?Hamburg, ?Breslau]. Palaearctic: Austria, Belarus, Belgium, China, Czech, Denmark, Estonia, Finland, France, Germany, Hungary, Ireland, Italy, Kazakhstan, Latvia, Lithuania, Luxembourg, Mongolia, Netherlands, Norway, Poland, Romania, Russia (Altai Rep., Arkhangelsk, Blagoveshchensk, Buryatia, Irkutsk, Kamchatka, Karelia, Komi, Krasnodar, Krasnoyarsk, Kuril Is., Leningrad, Lipetsk, Magadan, Moscow, Murmansk, Novgorod, Novosibirsk, Perm, Pskov, Sakhalin, Ural, Vladivostok, Vologda, Voronezh, Yakutia, Yaroslavl), Serbia, Slovakia, Sweden, Switzerland, UK, Ukraine (Kherson, Carpathians); Nearctic: Canada (Yukon), USA (Alaska).

\section{Dolichopus pennatus Meigen, 1824}

MATERIAL. Piy-Khem Kozhuun, Uyuk River floodplain at Uyuk village. $52.073^{\circ} \mathrm{N}, 94.043^{\circ} \mathrm{E}, 794 \mathrm{~m}$ a.s.l., 30 vi -5 vii 2017, O. Kosterin, 2 $\delta, 1$; Kaa-Khem Kozhuun, $18 \mathrm{~km} \mathrm{E}$ of Kyzyl, Serebryanka River in the Kaa-Khem River left bank floodplain at the bridge, larch/poplar forest, $51.701^{\circ} \mathrm{N}, 94.707^{\circ} \mathrm{E}, 638 \mathrm{~m}$ a.s.l., 6 vii 2017, O. Kosterin, $10^{\prime}$; Tes-Khem Kozhuun, Mazhalyg River, $880 \mathrm{~m}, 50.98^{\circ} \mathrm{N}, 95.18^{\circ} \mathrm{E}, 4$ vii 2017, N. Vikhrev, $1 \delta^{\wedge}$; $16 \mathrm{~km}$ SWS of Samagaltay village, the Tes Khem River right floodplain, grassy poplar forest, $50.470^{\circ} \mathrm{N}, 94.892^{\circ} \mathrm{E}, 1001 \mathrm{~m}$ a.s.l., 4 vii 2017, O. Kosterin, $4{ }^{\wedge}$.

DISTRIBUTION. Type locality: not given. Palaearctic: Austria, Belarus, Belgium, Bosnia and Herzegovina, Bulgaria, Czech, Denmark, Estonia, Finland, France, Georgia, Germany, Hungary, Ireland, Italy, Latvia, Lithuania, Mongolia, Netherlands, Norway, Poland, Portugal, Romania, Russia (Adygea, Alania, Altai Rep., Altai Terr., Arkhangelsk, Blagoveshchensk, Chechnya, Irkutsk, Kabardino-Balkaria, Kamchatka, Karachai-Cherkessia, Karelia, Khabarovsk, Krasnodar, Krasnoyarsk, Kuril Is., Leningrad, Magadan, Moscow, Murmansk, Novgorod, Pskov, Sakhalin, Tatarstan, Vladivostok, Vologda, Voronezh, Yakutia), Slovakia, Spain, Sweden, Switzerland, Turkey (Kars), UK, Ukraine.

\section{Dolichopus plumipes (Scopoli, 1763)}

MATERIAL. Piy-Khem Kozhuun, Uyuk River floodplain at Uyuk village. $52.073^{\circ} \mathrm{N}, 94.043^{\circ} \mathrm{E}, 794 \mathrm{~m}$ a.s.l., 30 vi -5 vii 2017, O. Kosterin, 5 $0^{\lambda}$, ; ; Tes-Khem Kozhuun, a pass through the Eastern Tannu-Ola Mts., grassy seepage in spruce taiga patch, $50.621^{\circ} \mathrm{N}, 95.193^{\circ} \mathrm{E}, \sim 1440 \mathrm{~m}$ a.s.l., 4 vii 2017 , O. Kosterin, $50^{\circ}$, 2 ; ; Tes-Khem River, $1000 \mathrm{~m}, 50.47^{\circ} \mathrm{N}, 94.91^{\circ} \mathrm{E}, 4$ vii 2017, N. Vikhrev, $20^{\circ}$; Uyuk River, 800 m, 52.07 N, $94.04^{\circ} \mathrm{E}, 1-3$ vii 2017, N. Vikhrev, $1 \mathrm{o}^{\circ}$.

DISTRIBUTION. Type locality: Slovenija: "Carnioliae indigena". Palaearctic: Afghanistan, Austria, Belarus, Belgium, Bosnia and Herzegovina, Bulgaria, China (Heilongjiang, Hebei, Henan, Shanxi, Inner Mongolia, Xinjiang, Qinghai, Xizang), Croatia, Czech, Denmark, Estonia, Finland, France, Georgia, Germany, Greece, Iceland, Italy, Hungary, Kazakhstan, Latvia, Lithuania, Mongolia, Netherlands, Norway, Poland, Romania, N Russia (Arkhangelsk, Karelia, Komi, Leningrad, Murmansk, Nenetsia, Novgorod, Pskov, Vologda), C Russia (Belgorod, Kirov, Mari El Republic, Nizhnii Novgorod, Moscow, Lipetsk, Perm, Tatarstan, Voronezh), S Russia (Adygea, Kabardino-Balkaria, Karachai-Cherkessia, Krasnodar, N Ossetia, Rostov), E Russia (Chukotka, Irkutsk, Kamchatka, Khabarovsk, Koryakia, Novosibirsk, Tomsk, Tyumen, Vladivostok), Slovakia, Spain, Sweden, Switzerland, Turkey (Tur Lake, Kackar Mountains, Rize), UK, Ukraine (Cherkasy, Kharkiv, Kiev, Lviv, Ternopil), Uzbekistan; Nearctic: Canada, USA, Greenland; Neotropical: Mexico; Oriental: China, India (Kashmir).

\section{Dolichopus pospelovi Smirnov, 1948}

MATERIAL. Uyuk River, $800 \mathrm{~m}, 52.07^{\circ} \mathrm{N}, 94.04^{\circ} \mathrm{E}, 1-3$ vii 2017, N. Vikhrev, 1 ภ. $^{\circ}$

DISTRIBUTION. Type locality: Russia: "Buchta Ta-Chingauz, river, Sudzukhinski reserve" [= Lazovski reserve]. Palaearctic: Russia (Vladivostok, Yakutia). 
Grichanov, I.Ya. New records of long-legged flies.... Acta Biologica Sibirica, 2018, 4(1), 6-16

\section{Dolichopus simius Parent, 1927}

MATERIAL. Piy-Khem Kozhuun, Uyuk River floodplain at Uyuk village. $52.073^{\circ} \mathrm{N}, 94.043^{\circ} \mathrm{E}, 794 \mathrm{~m}$ a.s.l., 30 vi - 5 vii 2017, O. Kosterin, $1 \delta^{\lambda}$; Uyuk River, 800 m, 52.07 N, 94.04 E, 1-3 vii 2017, N. Vikhrev, $1 \delta^{\text {. }}$.

DISTRIBUTION. Type locality: Russia: "Siberie: environs d'Irkutsk". Palaearctic: Russia (Altai Rep., Bashkortostan, Blagoveshchensk, Buryatia, Chita, Commander Is., Irkutsk, Kamchatka, Khabarovsk, Khakassia, Krasnoyarsk, Magadan, Moscow, Novosibirsk, Sakhalin, Sverdlovsk, Tomsk, Vladivostok, Yakutia), Mongolia, China (Heilongjiang, Inner Mongolia).

\section{Dolichopus urbanus Meigen, 1824}

MATERIAL. Piy-Khem Kozhuun, Uyuk River floodplain at Uyuk village. 52.073 N, $94.043^{\circ}$ E, $794 \mathrm{~m}$ a.s.l., 30 vi - 5 vii 2017, O. Kosterin, 2ᄋ; Kaa-Khem Kozhuun, 18 km E of Kyzyl, Serebryanka River in the Kaa-Khem River left bank floodplain at the bridge, larch/poplar forest, $51.701^{\circ} \mathrm{N}, 94.707^{\circ} \mathrm{E}, 638 \mathrm{~m}$ a.s.I., 6 vii 2017, O. Kosterin, $11 \delta^{\lambda}$, 5; Tes-Khem Kozhuun, $16 \mathrm{~km}$ SWS of Samagaltay village, the Tes Khem River right floodplain, grassy poplar forest, $50.470^{\circ} \mathrm{N}, 94.892^{\circ} \mathrm{E}, 1001 \mathrm{~m}$ a.s.l., 4 vii 2017, O. Kosterin, 10,1 .

DISTRIBUTION. Type locality: not given. Palaearctic: Austria, Belgium, Czech, Denmark, Estonia, Finland, France, Germany, Ireland, Italy, Latvia, Lithuania, Netherlands, Norway, Poland, Russia (Altai Rep., Arkhangelsk, Buryatia, Irkutsk, Kabardino-Balkaria, Karachai-Cherkessia, Karelia, Khantia-Mansia, Krasnodar, Krasnoyarsk, Leningrad, Moscow, Murmansk, Voronezh, S Ural), Slovakia, Sweden, Switzerland, UK.

\section{Gymnopternus Loew, 1857}

\section{Gymnopternus aerosus (Fallén, 1823)}

MATERIAL. Piy-Khem Kozhuun, Uyuk River floodplain at Uyuk village. $52.073^{\circ} \mathrm{N}, 94.043^{\circ} \mathrm{E}, 794 \mathrm{~m}$ a.s.l., $30 \mathrm{vi}-5 \mathrm{vii}$ 2017, O. Kosterin, 1 \% .

DISTRIBUTION. Type locality: not given [Sweden]. Palaearctic: Abkhazia, Austria, Belarus, Belgium, Bulgaria, Czech, Denmark, Estonia, Finland, France, Germany, Hungary, Ireland, Italy, Japan, Kazakhstan, Latvia, Lithuania, Mongolia, Netherlands, Norway, Poland, Romania, Russia (Adygea, Alania, Arkhangelsk, Kaliningrad, Karelia, Karachai-Cherkessia, Krasnodar, Leningrad, Lipetsk, Mordovia, Moscow, Murmansk, Novgorod, Pskov, Tatarstan, Voronezh, "Ural", Buryatia, Irkutsk, Khantia-Mansia, Vladivostok), Slovakia, Sweden, Tajikistan, UK, Ukraine (Chernovtsy, Kherson, Odessa, Uzhhorod); Oriental: Taiwan.

\section{Gymnopternus celer (Meigen, 1824)}

MATERIAL. Piy-Khem Kozhuun, Uyuk River floodplain at Uyuk village. 52.073 N, $94.043^{\circ}$ E, $794 \mathrm{~m}$ a.s.l., 30 vi - 5 vii 2017, O. Kosterin, 1 \%. Kaa-Khem Kozhuun, 18 km E of Kyzyl, Serebryanka River in the Kaa-Khem River left bank floodplain at the bridge, larch/poplar forest, $51.701^{\circ} \mathrm{N}, 94.707^{\circ} \mathrm{E}, 638 \mathrm{~m}$ a.s.I., 6 vii 2017, O. Kosterin, $1 \mathrm{~J}^{\mathrm{N}}, 4$..

DISTRIBUTION. Type locality: not given. Palaearctic: Austria, Belgium, Bosnia and Herzegovina, Bulgaria, Czech, Denmark, Estonia, Finland, France, Germany, Hungary, Ireland, Italy, Karelia, Kazakhstan, Latvia, Lithuania, Luxembourg, Netherlands, Norway, Poland, Romania, Russia (Altai Rep, Altai Terr., Buryatia, Krasnodar, Krasnoyarsk, Mordovia, Moscow, Novgorod, Novosibirsk, Pskov, Ryazan, Tatarstan, Ural, Voronezh), Serbia, Slovakia, Sweden, Switzerland, Turkey (Canakkale), UK, Ukraine (Chernovtsy, Kyiv, Lviv, Poltava, Uzhhorod).

\section{Hercostomus Loew, 1857}

\section{Hercostomus fulvicaudis (Haliday, 1851)}

MATERIAL. Kaa-Khem Kozhuun, 18 km E of Kyzyl, Serebryanka River in the Kaa-Khem River left bank floodplain at the bridge, larch/poplar forest, $51.701^{\circ} \mathrm{N}, 94.707^{\circ} \mathrm{E}, 638 \mathrm{~m}$ a.s.l., 6 vii 2017, O. Kosterin, 1 ; ; Tes-Khem Kozhuun, a pass through the Eastern Tannu-Ola Mts., grassy seepage in spruce taiga patch, $50.621^{\circ} \mathrm{N}, 95.193^{\circ} \mathrm{E}, \sim 1440 \mathrm{~m}$ a.s.l., 4 vii 2017 , O. Kosterin, 3 \%

DISTRIBUTION. Type locality: England. Palaearctic: Austria, Belgium, China, Czech, Estonia, France, Germany, Hungary, Iran, Netherlands, Poland, Romania, Russia (Lipetsk, Novosibirsk, Voronezh), Slovakia, Sweden, Switzerland, Tajikistan, Turkey (Afyonkarahisar, Kütahya), Turkmenistan, UK, Ukraine (Kherson, Odessa, Uzhhorod).

\section{Hydrophorus Fallén, 1823}

Hydrophorus sp.

MATERIAL. Tanda Kozhuun, salt Lake Dus-Khol, E bank, saline, 51.357 N, 94.446 E, 702 m a.s.l., 2 vii 2017, O. Kosterin, 3 3,6 ; Tes-Khem Kozhuun, a pass through the Eastern Tannu-Ola Mts., grassy seepage in spruce taiga patch, $50.621^{\circ} \mathrm{N}, 95.193^{\circ} \mathrm{E}, 1440 \mathrm{~m}$ a.s.l., 4 vii 2017, O. Kosterin, 1 \%.

REMARKS. The material examined belongs most probably to an undescribed species.

\section{Medetera Fischer von Waldheim, 1819}

Medetera jacula (Fallén, 1823)

MATERIAL. Uyuk River, $800 \mathrm{~m}, 52.07^{\circ} \mathrm{N}, 94.04^{\circ} \mathrm{E}, 1-3$ vii 2017, N. Vikhrev, $3{ }^{\widehat{ }}$.

DISTRIBUTION. Type locality: Sweden: Scania. Palaearctic: Armenia; Austria, Azerbaijan; Belarus, Belgium, Czech, Denmark, Estonia, Finland, France, Georgia; Germany, Hungary, Iran, Ireland, Italy, N Kazakhstan, Latvia, Lithuania, 
Grichanov, I.Ya. New records of long-legged flies.... Acta Biologica Sibirica, 2018, 4(1), 6-16

Netherlands, Norway, Poland, Romania; Russia (Alania, Altai, Buryatia, Irkutsk, Chechnya, Crimea, Kabardino-Balkaria, Kaluga, Karelia, Krasnodar, Kursk, Leningrad, Mordovia, Moscow, Novgorod, Novosibirsk, Pskov, Rostov, Ryazan, Stavropol, Urals, Vologda, Voronezh, Yakutia); Slovakia, Sweden, Switzerland, Tunisia, Turkey (Antalya), UK, Ukraine (Kharkiv, Kherson, Luhansk, Odessa, Poltava).

\section{Medetera pallipes (Zetterstedt, 1843)}

MATERIAL. Dus-Khol salt Lake, $700 \mathrm{~m}, 51.36^{\circ} \mathrm{N}, 94.45^{\circ} \mathrm{E}, 2-5$ vii 2017, N. Vikhrev, $1 \delta^{\lambda}$.

DISTRIBUTION. Type locality: Scania, "in Ostrog ad Wadstena; Botnia orientali ad Johannis Ro prope Tormea" [Sweden; Denmark]. Palaearctic: Austria, Belgium, Czech, Denmark, Egypt, Estonia, Finland, France, Georgia, Germany, Greece, Hungary, Iran, Israel, Morocco, Netherlands, Norway, Poland, Romania, Russia (Adygea, Chechnya, Crimea, Kabardino-Balkaria, Karelia, Krasnodar, Leningrad, Novgorod, Pskov, Ryazan, Stavropol, Voronezh), Slovakia, Spain, Switzerland, Sweden, Turkey (Antalya, Hakkari, Kars), UK, Ukraine (Kherson). The easternmost point of distribution.

\section{Melanostolus Kowarz, 1884}

\section{Melanostolus longipilosus Negrobov, 1985}

MATERIAL. Kaa-Khem Kozhuun, 18 km E of Kyzyl, the Kaa-Khem River left bank floodplain at the bridge, larch/poplar forest, $51.706^{\circ} \mathrm{N}, 94.712^{\circ} \mathrm{E}, 639 \mathrm{~m}$ a.s.l., 3 vii 2017, O. Kosterin, 1 \%; Dus-Khol salt Lake, $700 \mathrm{~m}, 51.36^{\circ} \mathrm{N}, 94.45^{\circ} \mathrm{E}, 2-5$ vii

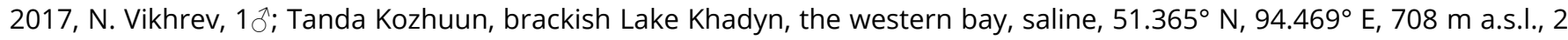
vii 2017, O. Kosterin, 1 ô.

ADDITIONAL MATERIAL. W Yakutia: env. Tumul, $60 \mathrm{~km} \mathrm{~N}$ vil. Suntar, Kyukei Lake, N 6243'38", E 11745'44",

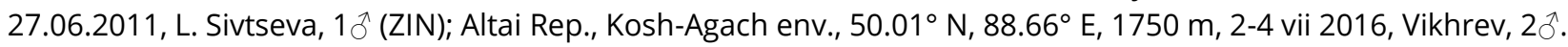

DISTRIBUTION. Type locality: Mongolia: [Altai Mts.,] Bajan-Olgij. Palaearctic: Mongolia. New for Russia.

REMARKS. The males from the Altai Republic fully correspond to the original description of M. longipilosus (Negrobov, 1985). The material from Tyva and a male from Yakutia have some fine differences from the typical form and can represent an undescribed species.

\section{Neurigona Rondani, 1856 \\ Neurigona davshinica Negrobov, 1987}

MATERIAL. Kyzyl env., poplar forest, 51.7 N, 94.7 E, 5-7 vii 2017, N. Vikhrev, $1 \delta^{\Uparrow}$.

DISTRIBUTION. Type locality: Russia: Barguzin Reserve, Davshe. Palaearctic: Russia (Buryatia).

\section{Rhaphium Meigen, 1803 \\ Rhaphium sp.}

MATERIAL. Kaa-Khem Kozhuun, 18 km E of Kyzyl, the Kaa-Khem River left bank floodplain at the bridge, larch/poplar forest, $51.706^{\circ} \mathrm{N}, 94.712^{\circ} \mathrm{E}, 639 \mathrm{~m}$ a.s.I., 3 vii 2017, O. Kosterin, 1 ; ; Tes-Khem Kozhuun, 16 km SWS of Samagaltay village, the Tes Khem River right floodplain, grassy poplar forest, $50.470^{\circ} \mathrm{N}, 94.892^{\circ} \mathrm{E}, 1001 \mathrm{~m}$ a.s.l., 4 vii 2017, O. Kosterin, 1 ㅇ.

REMARKS. Sibling Rhaphium species are hardly distinguished by females.

\section{Scellus Loew, 1857}

Scellus gallicanus Becker, 1909

MATERIAL. Tes-Khem Kozhuun, a pass through the Eastern Tannu-Ola Mts., grassy seepage in spruce taiga patch, $50.621^{\circ} \mathrm{N}, 95.193^{\circ} \mathrm{E}, \sim 1440 \mathrm{~m}$ a.s.l., 4 vii 2017, O. Kosterin, $1 \delta^{7}$.

DISTRIBUTION. Type locality: France: Lautaret. Palaearctic: China, France, Mongolia, Russia (Altai Rep., Murmansk, Sayan Mnts., Irkutsk, Yakutia).

\section{Sympycnus Loew, 1857}

\section{Sympycnus changaicus Negrobov 1973}

MATERIAL. Uyuk River, 800 m, 52.07 N, 94.04 E, 1-3 vii 2017, N. Vikhrev, 3 ${ }^{\circ}$; Piy-Khem Kozhuun, Uyuk River floodplain at Uyuk village. $52.073^{\circ} \mathrm{N}, 94.043^{\circ} \mathrm{E}, 794 \mathrm{~m}$ a.s.l., $30 \mathrm{vi}-5$ vii 2017, O. Kosterin, 1 \%.

DISTRIBUTION. Type locality: Mongolia: "Archangaj aimak, Gebirgspas Egijn davaa". Palaearctic: Mongolia, Russia (Altai Rep., Buryatia, Chita, Yakutia).

\section{Sympycnus pulicarius (Fallén, 1823)}

MATERIAL. Kaa-Khem Kozhuun, 18 km E of Kyzyl, Serebryanka River in the Kaa-Khem River left bank floodplain at the bridge, larch/poplar forest, $51.701^{\circ} \mathrm{N}, 94.707^{\circ} \mathrm{E}, 638 \mathrm{~m}$ a.s.I., 6 vii 2017, O. Kosterin, 3 $0^{2}, 3$; ; Tes-Khem Kozhuun, a pass through the Eastern Tannu-Ola Mts., grassy seepage in spruce taiga patch, $50.621^{\circ} \mathrm{N}, 95.193^{\circ} \mathrm{E}, \sim 1440 \mathrm{~m}$ a.s.l., 4 vii 2017 , O. Kosterin, 1 \%.

DISTRIBUTION. Type locality: not given [Sweden]. Nearctic: USA: California; Palaearctic: Andorra, Austria, Azerbaijan, Belgium, Bosnia and Herzegovina, Bulgaria, Croatia, Czech, Denmark, Estonia, Finland, France, Georgia, Germany, Greece, Hungary, Iran, Ireland, Italy, Kazakhstan, Kyrgyzstan, Latvia, Lithuania, Luxembourg, Macedonia, Moldova, Mongolia, Netherlands, Norway, Poland, Portugal, Romania, Russia (Alania, Altai Rep., Altai Ter., Buryatia, Chechnya, Chelyabinsk, 
Grichanov, I.Ya. New records of long-legged flies.... Acta Biologica Sibirica, 2018, 4(1), 6-16

Crimea, Kabardino-Balkaria, Kaliningrad, Karachai-Cherkessia, Karelia, Khakassia, Krasnodar, Krasnoyarsk, Leningrad, Lipetsk, Moscow, Murmansk, Novgorod, Novosibirsk, Pskov, Stavropol, Svedlovsk, Tambov, Tatarstan, Voronezh, Yakutia), Serbia, Slovakia, Spain incl. Canary Islands, Sweden, Switzerland, Tajikistan, Turkey (Aydin, Bolu, Mugla, Van), UK, Ukraine (Kherson), Uzbekistan.

\section{Tachytrechus Haliday, 1851}

Tachytrechus vinogradovi Stackelberg, 1941

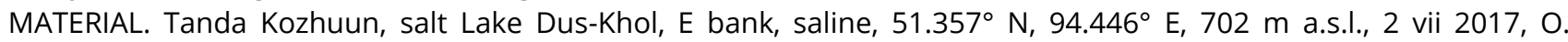

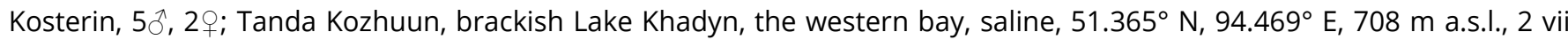
2017, O. Kosterin, $1 \hat{\jmath}, 1$ +

DISTRIBUTION. Type locality: Russia: "Transbaikalien, Ivan-See bei Tshita". Palaearctic: Kazakhstan, Mongolia, Russia (Buryatia, Chita).

\section{Thinophilus Wahlberg, 1844 \\ Thinophilus sp.}

MATERIAL. Tanda Kozhuun, salt Lake Dus-Khol, E bank, saline, 51.357 N, $94.446^{\circ} \mathrm{E}, 702 \mathrm{~m}$ a.s.l., 2 vii 2017 , O. Kosterin, 19 $\hat{\circ}$, 20\%; Tanda Kozhuun, brackish Lake Khadyn, the western bay, saline, $51.365^{\circ} \mathrm{N}, 94.469^{\circ} \mathrm{E}, 708 \mathrm{~m}$ a.s.l., 2 vii

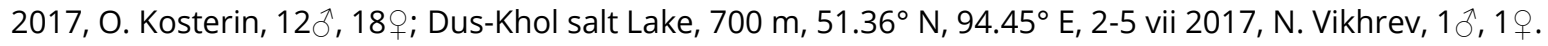

REMARKS. The material examined belongs most probably to an undescribed species.

\section{Thinophilus flavipalpis (Zetterstedt, 1843)}

MATERIAL. Tanda Kozhuun, salt Lake Dus-Khol, E bank, saline, $51.357^{\circ} \mathrm{N}, 94.446^{\circ} \mathrm{E}, 702 \mathrm{~m}$ a.s.l., 2 vii $2017, \mathrm{O}$.

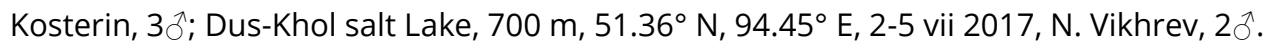

DISTRIBUTION. Type locality: Sweden: Gottlandia, Bursviken. Palaearctic: Austria, Azerbaijan, Belgium, Bulgaria, N China, Croatia, Czech, Denmark, Egypt, Estonia, Finland, France, Germany, Greece (Crete, North Aegean), Hungary, Iran, Israel, Italy, Kazakhstan, Kyrgyzstan, Moldova, Mongolia, Morocco, Netherlands, Poland, Portugal, Romania, S Russia (Astrakhan, Crimea, Krasnodar, Rostov), Spain, Sweden, Syria, Turkey (Ankara), UK, Ukraine (Kherson, Odessa); Oriental: China.

\section{Thinophilus setosus Negrobov, 1979}

MATERIAL. Tanda Kozhuun, salt Lake Dus-Khol, E bank, saline, $51.357^{\circ} \mathrm{N}, 94.446^{\circ} \mathrm{E}, 702 \mathrm{~m}$ a.s.l., 2 vii 2017 , O.

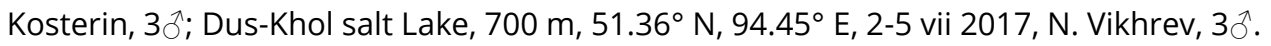

DISTRIBUTION. Type locality: Mongolia: Bajan-Chongorskij aimak, am Nordufer des Orog-Nuursees. Palaearctic: Mongolia. New for Russia.

REMARKS. At present, it is not possible to identify five females collected together with closely related Thinophilus flavipalpis and T. setosus.

\section{Thrypticus Gerstaecker, 1864}

Thrypticus bellus Loew, 1869

MATERIAL. Dus-Khol salt Lake, $700 \mathrm{~m}, 51.36^{\circ} \mathrm{N}, 94.45^{\circ} \mathrm{E}, 2-5$ vii 2017, N. Vikhrev, $1 \delta^{\wedge}$; Tanda Kozhuun, salt Lake DusKhol, E bank, saline, $51.357^{\circ} \mathrm{N}, 94.446^{\circ} \mathrm{E}, 702 \mathrm{~m}$ a.s.l., 2 vii 2017, O. Kosterin, 1 \%.

DISTRIBUTION. Type locality: England: Kew. Afrotropical: DR Congo, Kenya, Ethiopia, Senegal, South Africa, St. Helena, Tanzania. Palaearctic: Abkhazia, Armenia, Austria, Azerbaijan, Belgium, Bosnia and Herzegovina, Bulgaria, China, Croatia, Czech, Denmark, Egypt, Finland, France, Germany, Greece, Hungary, Iran, Ireland, Israel, Golan Heights, Italy, Kazakhstan, Lithuania, Morocco, Netherlands, Poland, Romania, Russia (Krasnoyarsk, Leningrad, Novosibirsk, Rostov, Vladivostok, Voronezh), Slovakia, Spain, Sweden, Switzerland, Turkey (Erzurum, Kars, Mugla), UK, Ukraine (Odessa).

\section{Conclusion}

As a result of this study, a new material of Dolichopodidae was identified, and a check-list of Tyvan species was compiled. The present research gives new records, including 34 species found for the first time in the Republic, and two probably undescribed species. Asyndetus longicornis is a new species for Siberia; Mongolian Melanostolus longipilosus and Thinophilus setosus are found for the first time in Russia. In total, 16 genera and 41 (including three unnamed) species are known from Tyva. Almost all species were collected along banks of rivers and lakes (see Figs. 1-6); nevertheless, Medetera and Neurigona species inhabited tree trunks (N.E. Vikhrev, pers. comm.).

Most collected species are widespread across the Palaearctic Region. At present, the Tyva Republic is the easternmost area of distribution for the dendrophilous Medetera pallipes. The following twelve species are not known in Europe: Dolichopus calceatus, D. galeatus, D. griseifacies, D. pospelovi, D. selivanovae, Hydrophorus zaitzevianus, Medetera asiatica, Melanostolus longipilosus, Neurigona davshinica, Sympycnus changaicus, Tachytrechus vinogradovi and Thinophilus setosus. 
Grichanov, I.Ya. New records of long-legged flies.... Acta Biologica Sibirica, 2018, 4(1), 6-16

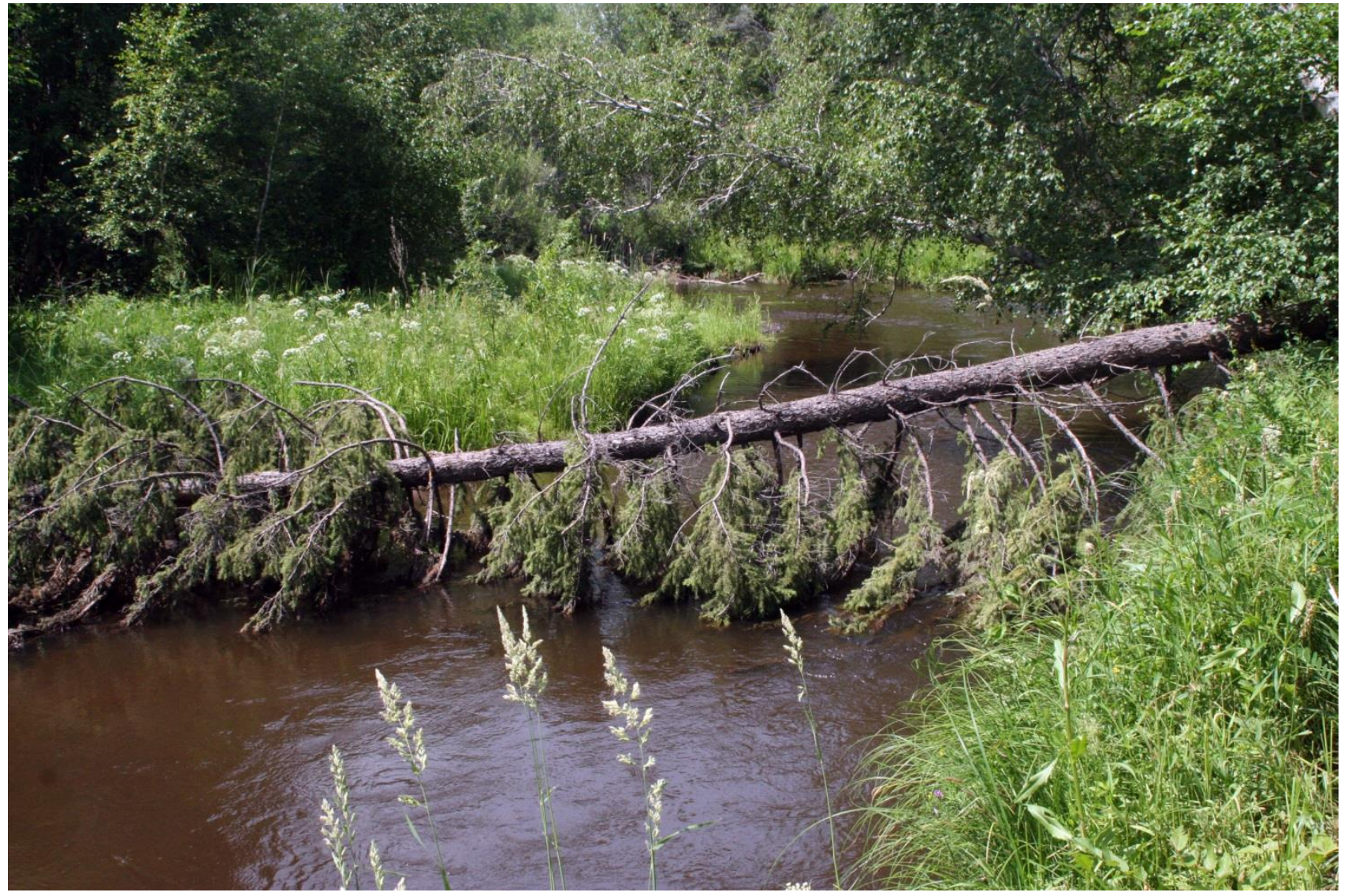

Fig. 1. Uyuk River. Piy-Khem Kozhuun, 30 June 2017.

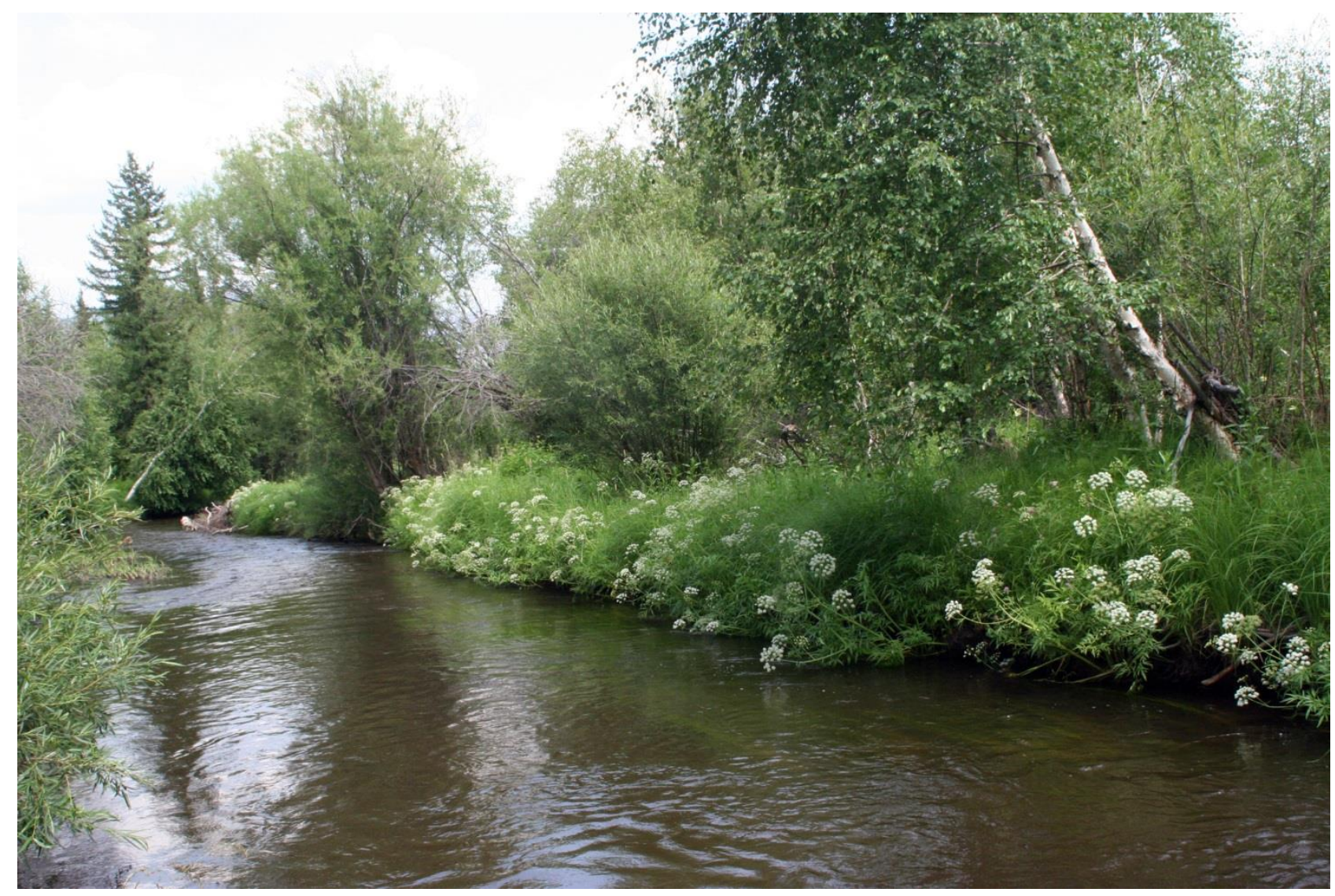

Fig. 2. Uyuk River. Piy-Khem Kozhuun, 30 June 2017. 
Grichanov, I.Ya. New records of long-legged flies.... Acta Biologica Sibirica, 2018, 4(1), 6-16

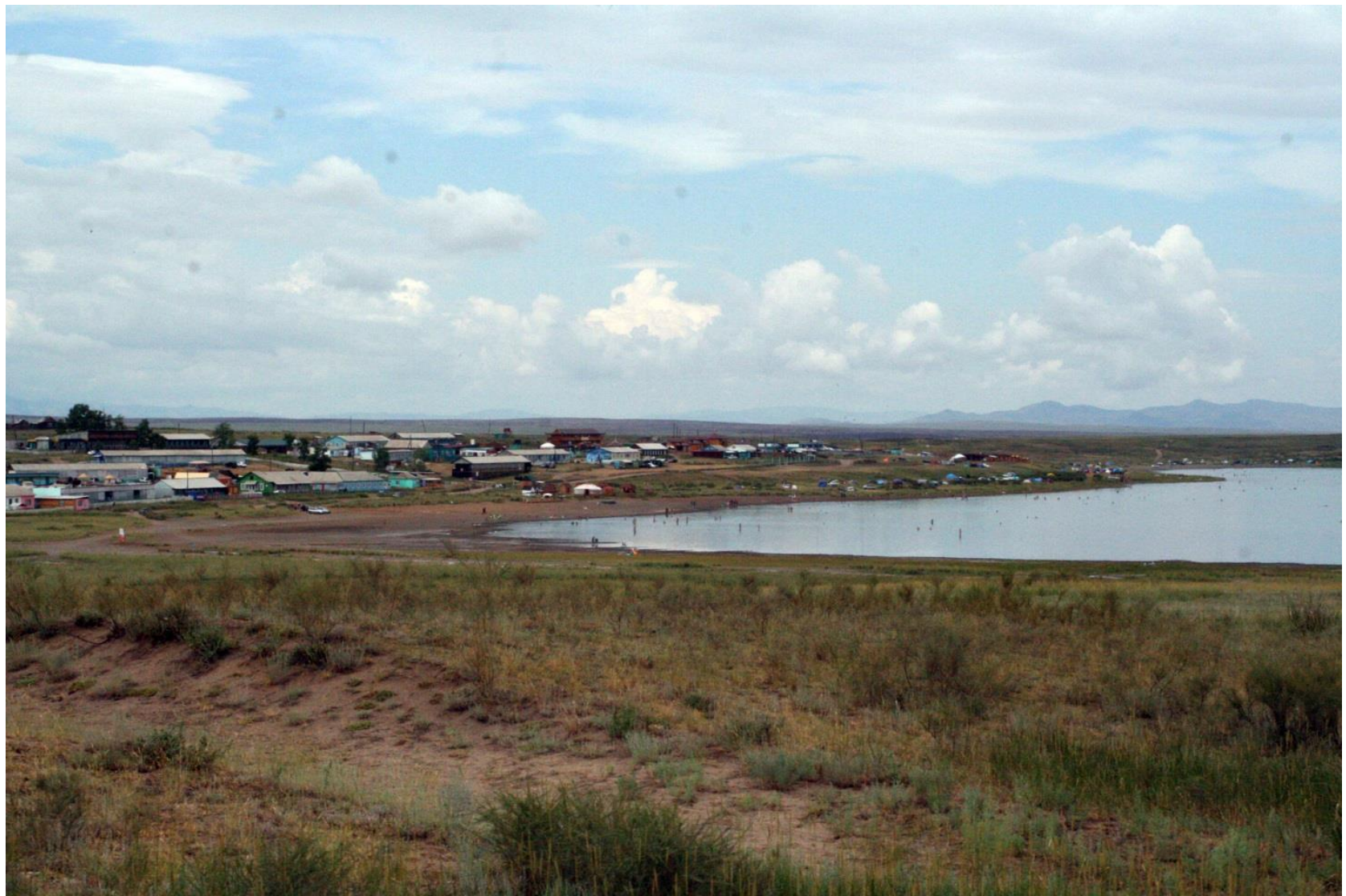

Fig. 3. Salt Lake Dus-Khol. Tanda Kozhuun, 2 July 2017.

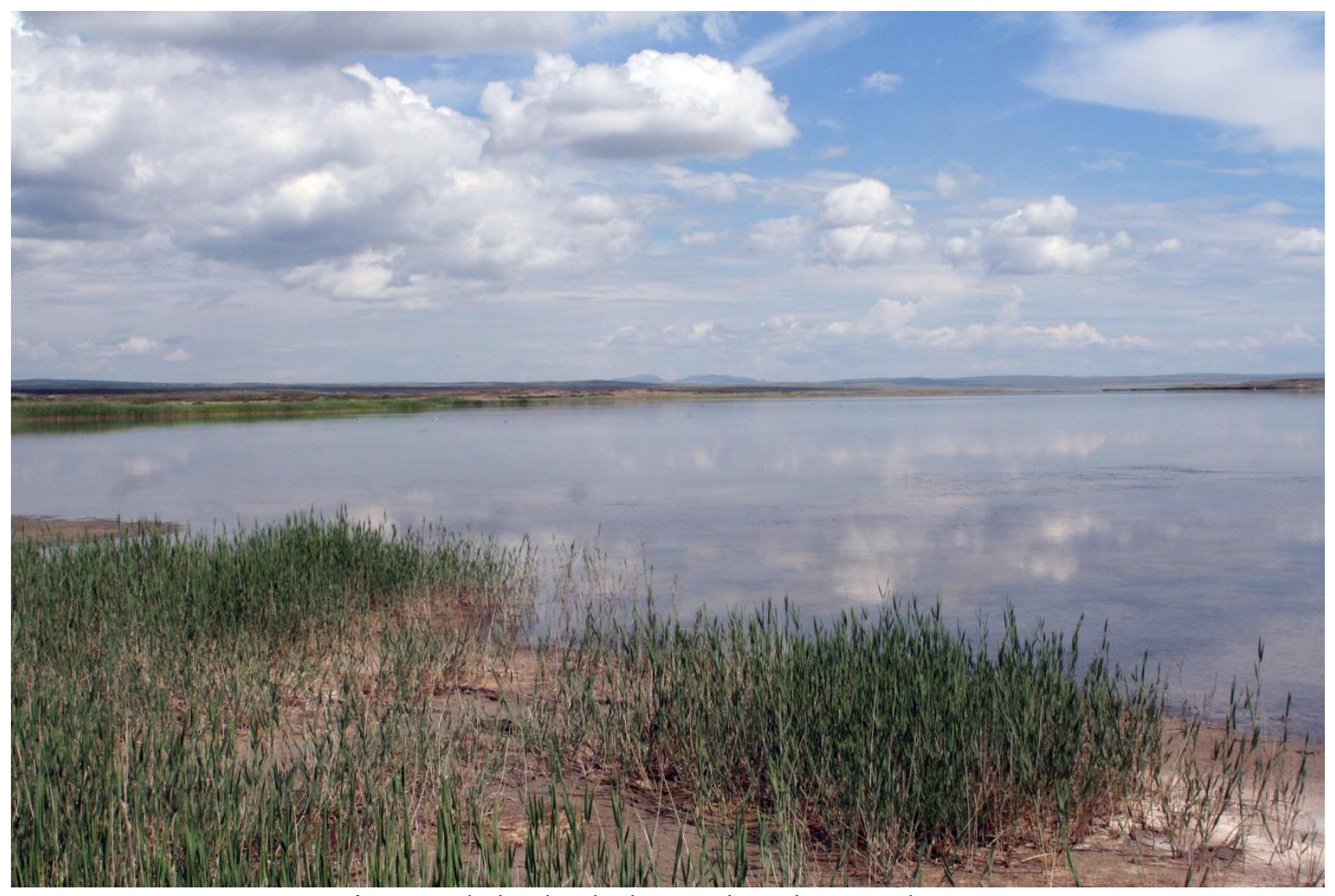

Fig. 4. Brackish Lake Khadyn. Tanda Kozhuun, 2 July 2017. 


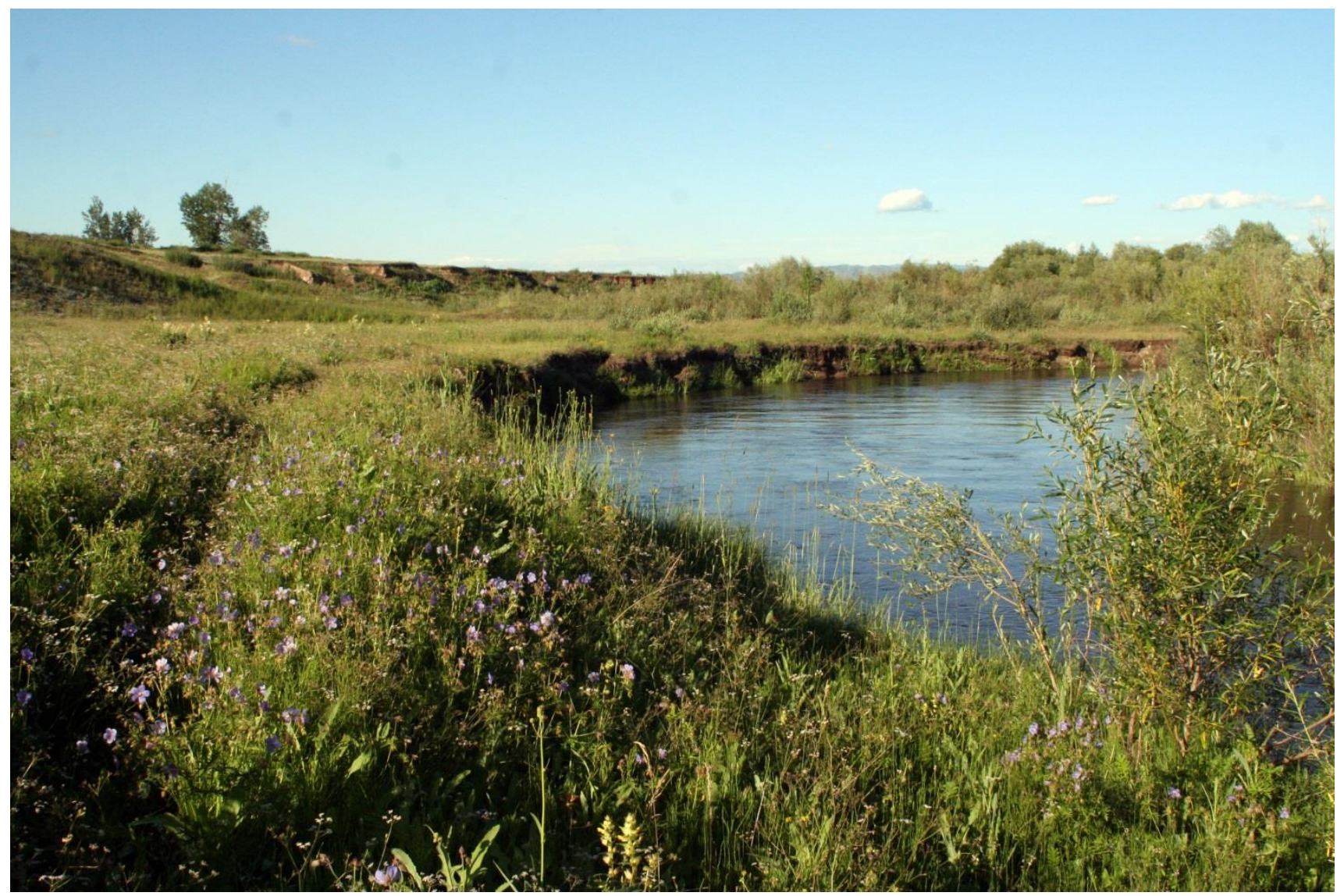

Fig. 5. Mazhalyg River. Tanda Kozhuun, 4 July 2017.

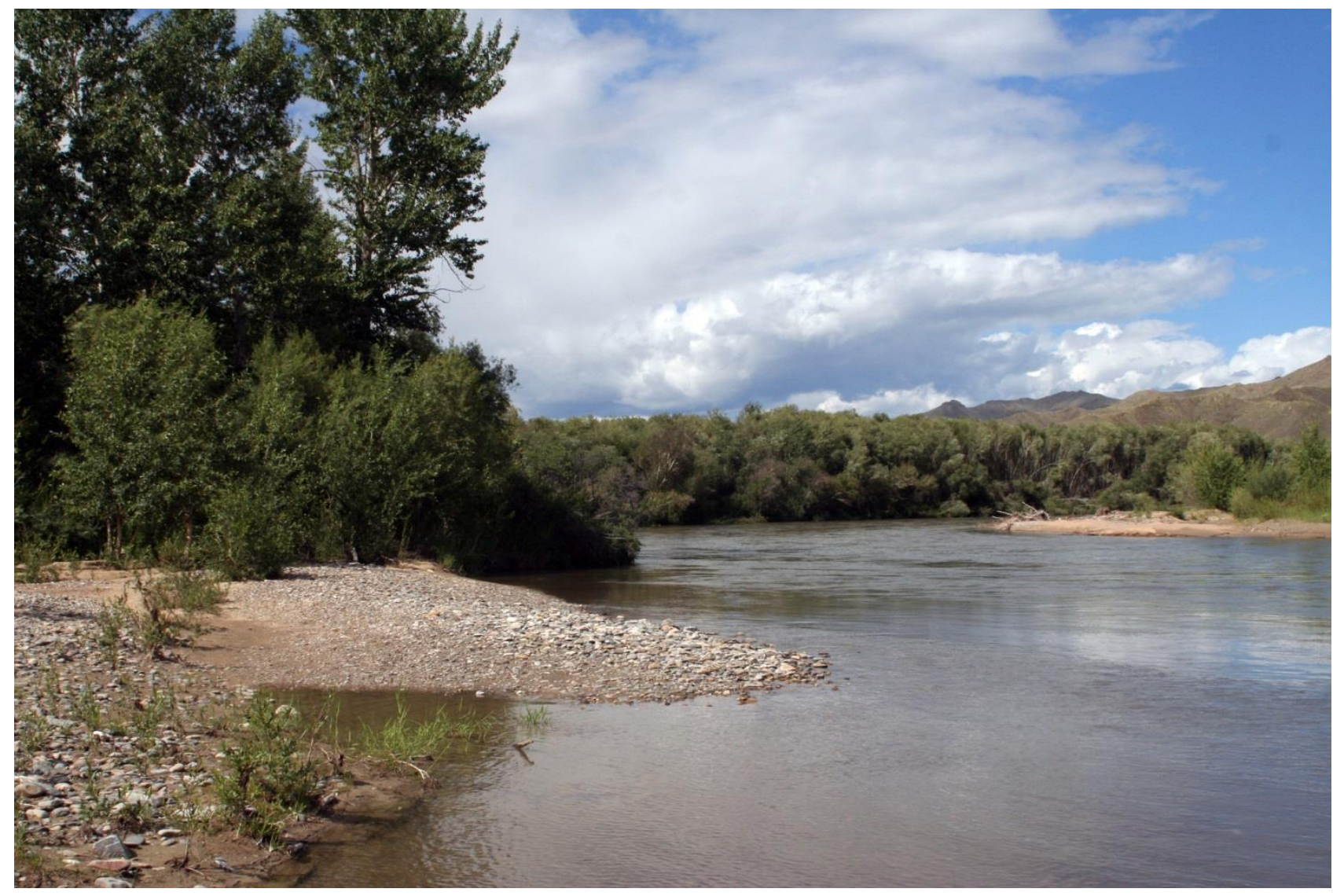

Fig. 6. Tes-Khem River. Tes-Khem Kozhuun, 4 July 2017.

\section{Acknowledgments}

I am grateful to collectors of the material Dr. N.E. Vikhrev, M. Yanbulat (Moscow) and Dr. O.E. Kosterin (Novosibirsk). Drs. O.P. Negrobov (Voronezh), N.E. Vikhrev and O.E. Kosterin kindly commented on earlier drafts of the manuscript. 


\section{References}

Grichanov, I.Ya. (2017). Alphabetic list of generic and specific names of predatory flies of the epifamily Dolichopodoidae (Diptera). Second edition. St.Petersburg: VIZR, 1-563. (Plant Protection News Supplements 23).

Negrobov, O.P. (1979). Family Dolichopodidae (Diptera) of the fauna of the USSR. I. Subfamilies Dolichopodinae and Medeterinae. Entomologicheskoe Obozrenie, 58(3), 646-659. (In Russian).

Negrobov, O.P. (1985). A revision of the Palaearctic species of the genus Melanostolus Kowarz (Diptera: Dolichopodidae). In: Narchuk, E.P., Zlobin, V.V. (Eds.). Diptera (Insecta) of the fauna of the USSR and their significance in ecosystems. Leningrad: Akademiya Nauk SSSR, 1984, 81-85 (in Russian).

Negrobov, O.P., Barkalov, A.V. (2010). New species of the genus Dolichopus (Diptera, Dolichopodidae) from mountains of Altai and Tuva. Zoologicheskii zhurnal, 89(11), 1361-1365 (in Russian).

Negrobov, O.P., Barkalov, A.V., Selivanova, O.V. (2010). New data on the fauna of the family Dolichopodidae (Diptera) from Russia, with a description of a new species of the genus Argyra Mcq. Euroasian Entomological Journal, 9(3), 522-524.

Negrobov, O.P., Selivanova, O.V., Maslova, O.O., Chursina M.A. (2013). Check-list of predatory flies of the family Dolichopodidae (Diptera) in the fauna of Russia. In: Grichanov, I.Ya., Negrobov, O.P. (Eds.). Fauna and taxonomy of Dolichopodidae (Diptera). Collection of papers. St.Petersburg: VIZR RAAS (Plant Protection News Suppl.), 47-93. Available from: https://archive.org/details/GrichanovNegrobovDolichopodidae2013 (accessed 30 August 2017).

Negrobov, O.P., Stackelberg, A.A. (1969). Family Dolichopodidae. In: Bei-Bienko, G.Ya. (Ed.). Keys to the Fauna of Euriopean Parts of USSR, 5(1). Leningrad: Nauka: 670-751 (in Russian).

Stackelberg, A.A. (1930). Dolichopodidae. In: Lindner, E. Die Fliegen der palearktischen Region. Bd. IV, 5, 29. Lief. 59, 1 64.

Yanovskii, V.M. (1976) Entomophages of trunk pests in Tuva ASSR and Mongolian People's Republic. In: Problems in Population Dynamics of the Insect Pests of Taiga Forests. Krasnoyarsk: Izdatel'stvo Sibirskogo Otdeleniya Akademii Nauk SSSR, 42-56 (in Russian).

\section{Citation:}

Grichanov, I.Ya. (2018). New records of long-legged flies (Diptera, Dolichopodidae) from Tyva Republic (Russia, Siberia). Acta Biologica Sibirica, 4 (1), 6-16.

Submitted: 28.11.2017. Accepted: 05.02.2018

cross ref http://dx.doi.org/10.14258/abs.v3i3.2184.3921

(C) 2018 by the authors. Submitted for possible open access publication under the terms and conditions of the Creative Commons Attribution (CC BY) license (http://creativecommons.org/licenses/by/4.0/). 